\title{
Evaluation of species diversity among Indian clinical Schizophyllum commune using matrix-assisted laser desorption ionization-time of flight mass spectrometry and MLST
}

\author{
Pradeep Kumar Singh ${ }^{1}$, Jacques F. Meis ${ }^{2}$, Anuradha Chowdhary ${ }^{1 *}$ \\ ${ }^{1}$ Department of Medical Mycology, Vallabhbhai Patel Chest Institute, University of Delhi, Delhi, India; ${ }^{2}$ Radboudumc/CWZ, \\ Nijmegen, Netherlands
}

\section{Objectives}

$>$ Schizophyllum is an important plant and animal pathogen in the genus basidiomycetes.

$>$ Schizophyllum commune is the best-known species causing allergic and invasive mycoses since 1950.

$>$ Key morphological features necessary for the recognition of basidiomycetes fungi are the presence of clamp connections on hyphae and the development of fruiting bodies.

$>$ However, the final species identification needs confirmation by molecular methods.

> The aim of this study was to determine the species diversity among Indian clinical isolates of Schizophyllum using multilocus phylogenetic analysis and matrix-assisted laser desorption ionization-time of flight mass spectrometry (MALDI-TOF MS, Bruker Biotyper).

\section{Methods}

$>$ Isolates: Thirty-seven molecularly identified clinical isolates of $S$. commune were selected from the culture collection of Medical Mycology, V. P. Chest Institute, Delhi, India, to determine the species diversity along with the reference isolates of $S$. commune (CBS 333.85, CBS 579.83, CBS109426 and CBS10320) and S. radiatum (CBS 301.32).

$>$ MLST: The markers used for sequencing were the internal transcribed spacer (ITS), a portion of the nuclear large subunit (LSU) of ribosomal DNA, the RNA polymerase II second-largest subunit (RPB2), and the translation elongation factor $1 \alpha \quad(E F-1 \alpha)$ gene. The neighbor joining (NJ) phylogenetic tree was constructed using MEGA (v.6) software.

> MALDI-TOF MS: The isolates were also processed for identification and to upgrade the in-house database by creating the main spectrum (MSP) using MALDI-TOF MS.

\section{Results}

> The LSU, EF-1a, and RPB2 markers showed consistency and concatenated sequences were analyzed. The analyses revealed two main clades in the phylogenetic tree.

$>$ One clade included all the Indian and CBS reference strains of $S$. commune $(n=38)$, and the other clade included only reference strain of $S$. radiatum (CBS 301.32) used in the study.

$>$ The reference strains of $S$. umbrinum and $S$. fasciatum acted as outgroups since the genetic distance to the clades of $S$. radiatum and $S$. commune were elevated.

$>$ MALDI-TOF MS showed all the isolates as $S$. commune including $S$. radiatum reference strain as the current Bruker database does not contain MSPs for $S$. radiatum.

\section{Results}

MALDI-TOF MS correctly identified all the isolates after the upgradation of database.

MSP dendogram also showed two different clusters for $S$. commune and $S$. radiatum, which were in concordance with the NJ phylogenetic tree based on concatenated sequences.

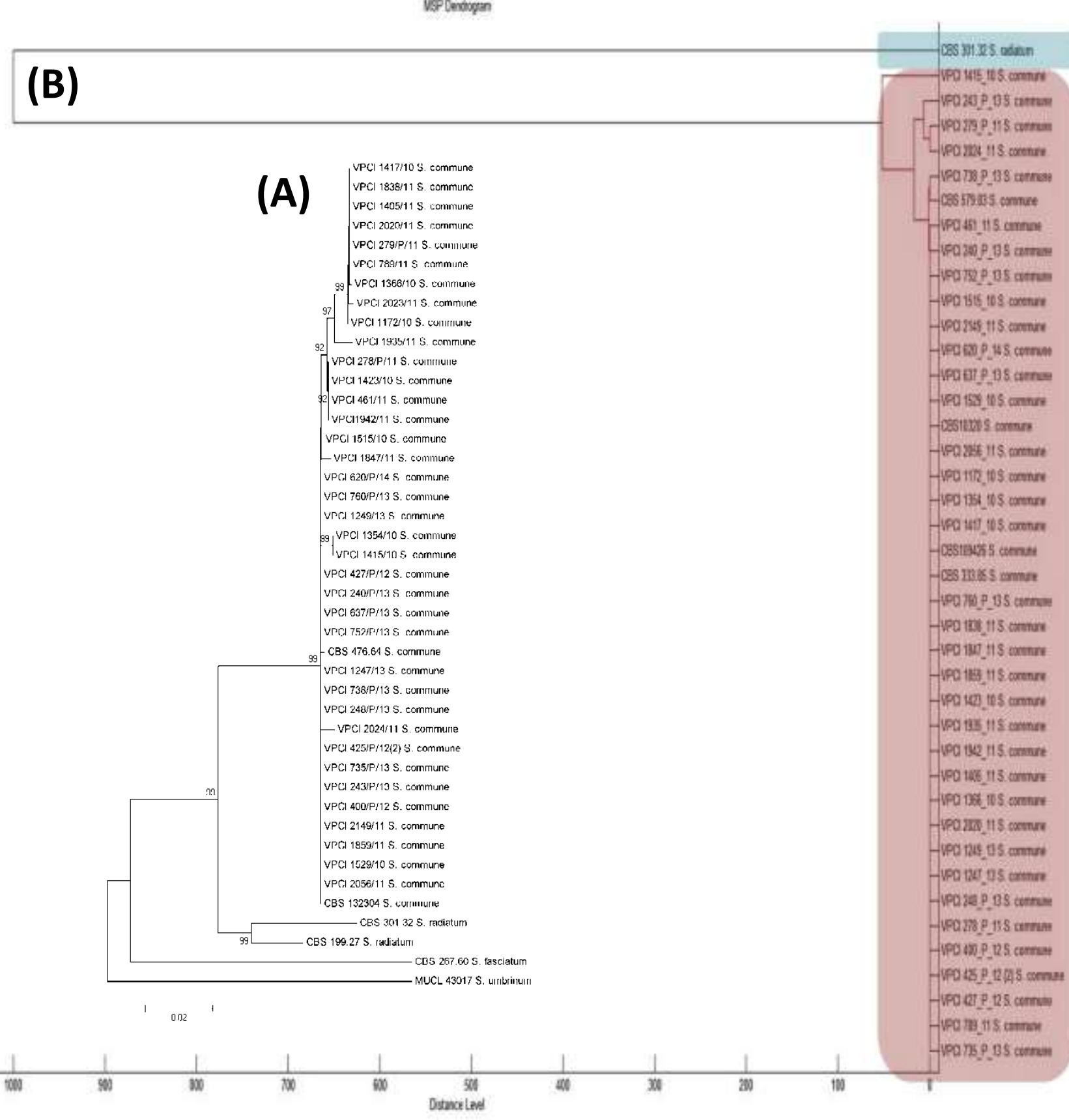

Figure: (A) Score-oriented dendrogram of the main spectra of Schizophyllum commune and S. radiatum; (B) Neighbour joining tree obtained from the combined LSU, EF-1a, and RPB2 sequences of the isolates. Bootstrap support values over $\mathbf{7 0}$ are indicated on the nodes.

\section{Conclusion}

$>$ The importance of Schizophyllum in the clinical setting has been demonstrated by the number of reported cases and strains isolated.

$>$ In Indian scenario, S. commune is the prevalent species in allergic and invasive pulmonary infections instead of $S$. radiatum which is most frequently recovered species from USA.

$>E F 1$ and RPB2 alone can differentiate between these two species of Schizophyllum and thus the two markers can be used for the identification of Schizophyllum species.

> MALDI-TOF MS plays an important role in rapid identification of Schizophyllum species as it takes only half an hour for the identification but it has limited database. Creation of in-house database can improve the identification rate drastically. 